\title{
Pancreatic ductal mucinous hyperplasia: distribution within the pancreas, and effect of variation in ampullary and pancreatic duct anatomy
}

\author{
T G ALLEN-MERSH \\ From the Charing Cross Hospital, London
}

SUMMARY The prevalence and amount (hyperplasia score) of ductal mucinous hyperplasia (DMH) were estimated in 12 predetermined areas taken from 102 pancreases obtained at necropsy from patients dying of non-pancreatic diseases. The mean prevalence of DMH was $18 \%(213 / 1206)$ per section and $63 \%(63 / 102)$ per pancreas. Different areas of the pancreas could be stratified by amount of DMH into 'high' (hyperplasia score >0.09), 'intermediate' $(0.06-0.09)$, and 'low' $(<0.06)$. There was no significant difference in either prevalence or amount of DMH with variation in ampullary or pancreatic ductal anatomy. There was significantly $(\mathbf{p}<0.05)$ less $\mathrm{DMH}$ adjacent to the accessory papilla when it was patent, compared with when it was not patent and the accessory duct communicated with the main pancreatic duct. The findings suggest that DMH is a proliferative response to exogenous agents which injure the pancreas, and that some areas of the pancreas are more vulnerable than others to this damage. There was no evidence that this injury was associated either with reflux, or with any particular variation in pancreatic duct anatomy. The association between DMH and occlusion of the accessory papilla may explain the susceptibility of pancreas divisum to pancreatitis.

Pancreatic ductal mucinous hyperplasia (DMH) is thought to be a hyperplastic and metaplastic response by pancreatic duct and acinar cells to a variety of proliferative stimuli.' It has been seen as a response to carcinogen exposure in experimental models of pancreatic neoplasia ${ }^{23}$ but it is also commonly found in chronic pancreatitis ${ }^{4}$ where its presence suggests a proliferative response to injury, and in association with corticosteroids' which are known to produce pancreatitis. $^{5}$

The increased prevalence of DMH which has been observed $^{16}$ in the head compared with the body of the pancreas could be explained if the stimulus resulting in DMH was produced by an agent which exerted a greater effect on the head than on the body of the pancreas. Possible sources of a stimulus which could produce such a differential effect include: (a) reflux of either bile, which may be promoted by certain

Address for correspondence: T G Allen-Mersh, MD FRCS, Department of Surgery, Charing Cross and Westminster Medical School, Fulham Palace Road, London W6 8RF.

Received for publication 4 May 1988. anatomical arrangements of the ampullary sphincter, ${ }^{7}$ or duodenal fluid ${ }^{8}$ into the pancreas. (b) Stasis of pancreatic juice because of decreased pancreatic drainage - affecting only that area of the pancreas drained by the blocked duct - as occurs in ventral unfused pancreas."

The purpose of the present study was to accurately identify the distribution of DMH within the pancreas, and to determine whether variation in amount or distribution of DMH could be attributed to variation in either ampullary or pancreatic duct anatomy.

\section{Methods}

Pancreas was obtained at necropsy, carried out within 48 hours of death, on patients who had died of non-pancreatic diseases. Preliminary experiments suggested that pancreas obtained within 48 hours of death from refrigerated cadavers was not autolysed on histological examination. The pancreas was removed in a block including pylorus, duodenum, distal bile duct, and spleen. The tip of the tail of the 
Table The duct classification was derived by combining the information obtained at the time of dye injection with that obtained from cutting sagittal slices through the pancreas

\begin{tabular}{lll}
\hline Duct category & Dye injection & Appearance of slices \\
\hline I & $\begin{array}{c}\text { Dye at both papillae. } \\
\text { main papilla first } \\
\text { Dye at both papillae, } \\
\text { accessory papilla first } \\
\text { II }\end{array}$ & $\begin{array}{c}\text { Main and accessory } \\
\text { duct stained } \\
\text { Main and accessory } \\
\text { duct stained } \\
\text { No staining of accessory } \\
\text { duct }\end{array}$ \\
III & $\begin{array}{c}\text { Dye at accessory papilla } \\
\text { only }\end{array}$ & $\begin{array}{c}\text { No staining of main } \\
\text { duct in head } \\
\text { IV }\end{array}$ \\
Dye at main papilla only & $\begin{array}{c}\text { Main and accessory } \\
\text { ducts stained }\end{array}$ \\
\hline
\end{tabular}
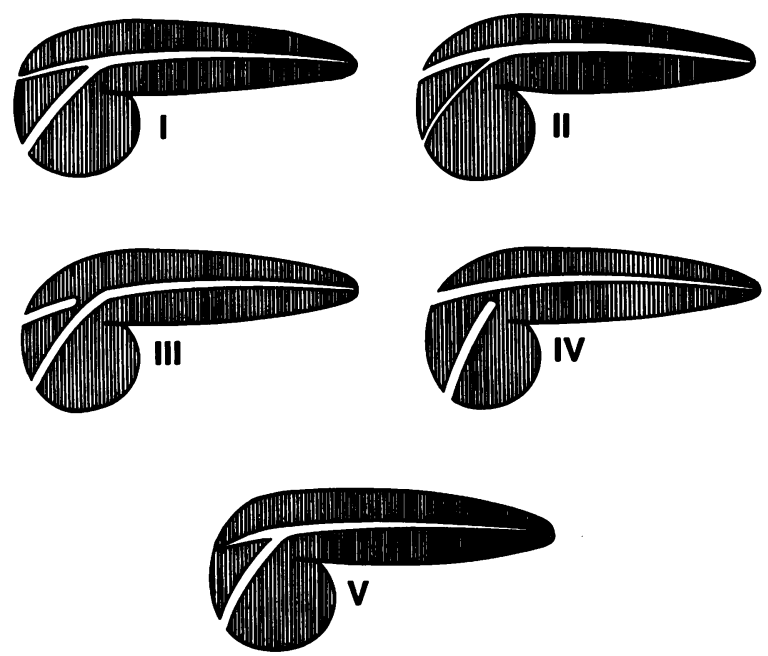

Fig. 1 Categories of duct anatomy to which the classification in the Table refers.

pancreas was then incised and a $1.1 \mathrm{~mm}$, od, Medicut cannula inserted into the pancreatic duct. Approximately $1 \mathrm{ml}$ of a solution of $1 \%$ crystal violet dye dissolved in $10 \%$ formalin was then injected. As the dye flowed along the pancreatic duct it imparted a ripple to the surface of the pancreas which allowed the injection rate to be slowed as dye approached the papilla. A final slow injection of $0.25 \mathrm{ml}$ caused dye to appear at the ampulla. The distance between the pancreatic duct opening and the tip of the papilla was then measured with dividers and classified as: $0 \mathrm{~mm}$, $<2 \mathrm{~mm}, 2-5 \mathrm{~mm}$, and $>5 \mathrm{~mm}$.

The pancreatic duct orifice was then pinched off with a pair of fine forceps and a further $1 \mathrm{ml}$ injected through the cannula at a pressure of approximately $200 \mathrm{mmHg}$. The duodenum proximal and ventral to the main papilla was scrutinised for dye emerging from the accessory papilla. Occasionally dye emerged first from the accessory papilla, in which

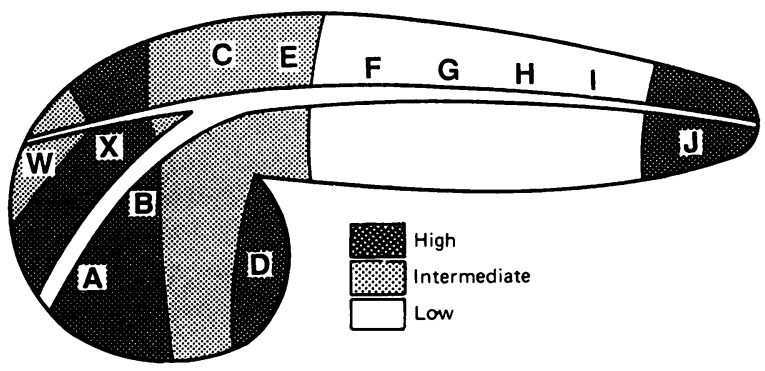

Fig. 2 Standard areas $(A-J, W$, and $X)$ from which tissue blocks were taken. It was possible to divide the pancreas into 'high', 'intermediate', or 'low' areas according to the amount of hyperplasia seen. There was a significant (paired t test $p<0.01$ ) difference between pancreas hyperplasia score in any 'high' area compared with any 'low' area within the same pancreas.

case this was pinched off and the main papilla was observed for the presence of dye after extra injection under pressure. The pancreas was pinned out and fixed in $10 \%$ buffered formalin for five days after which it was sagittally sliced at $1 \mathrm{~cm}$ intervals. Pancreatic ducts could be identified by the crystal violet stain in these sagittal slices. Frequently both main and accessory ducts were stained, but in some cases only one duct was outlined while in other cases both ducts were outlined in a specimen where dye had not emerged through both papillae. These findings suggested a functional classification of pancreatic anatomy (Table, Fig. 1).

Blocks of tissue for histological examination were taken (Fig. 2) from two sites in the embryologically 'ventral' component ${ }^{\text {"I }}$ of the pancreatic head (areas A, B), two sites in the embryologically 'dorsal' component of the pancreatic head (area W, X), the site of fusion of the two duct systems (area C), the uncinate process (area D), the pancreatic neck (area E), three sites in the pancreatic body (areas F, G, H), and two sites in the tail (areas I, J). The site of fusion of the dorsal and ventral embryological components of the pancreatic head" was usually evident in the sagittal slices (Fig. 3).

Sections were stained with haematoxylin and eosin, and the amount of DMH seen was scored using a graticule eyepiece and light microscope as previously described.' In brief, a score of 1 was given wherever an area of either ductular metaplasia or papillary ductal hyperplasia was seen, and a score of 2 where both were seen in the same field. This provided a hyperplasia score for the amount of $\mathrm{DMH}$ seen in one field. A hyperplasia 'score' for the entire pancreas was obtained by adding the scores for each graticule area and dividing by the number of areas examined.' One hundred and two specimens were examined. In each specimen, the amount of DMH 
seen in one section (mean area $148.8 \mathrm{~mm}^{2}$, range $21 \cdot 2-190 \cdot 8 \mathrm{~mm}^{2}$ ) taken from each of the 12 defined areas (Fig. 2) was recorded.

Hyperplasia score was not normally distributed and so the significance of differences in hyperplasia score was estimated by a non-parametric (Wilcoxon's) test. Where hyperplasia scores within the same pancreas were compared, a paired Student's $t$ test was appropriate because the distribution of difference between two hyperplasia scores within a pancreas was approximately normal. Pearson correlation was used to correlate hyperplasia scores in different areas of the same pancreas. Prevalence of hyperplasia was compared in different areas by $2 \times 2$ contingency table. Results were analysed by computer using SPSS. " Complete information about every case was not available; therefore the number of cases in each analysis varied slightly.

\section{Results}

PREVALENCE AND DISTRIBUTION OF DMH

WITHIN THE PANCREAS

Ductal mucinous hyperplasia was found in $62 \%(63 /$. 102) of pancreases examined. Mean prevalence of DMH per section was $18 \%$ (213/1206). Eighteen sections were not evaluable because of tissue autolysis. Ductal mucinous hyperplasia was not uniformly distributed throughout the pancreas (Fig. 2). The pancreas could be divided by hyperplasia score into: 'high' (hyperplasia score >0.09), 'intermediate' (hyperplasia score 0.09-0.06), and 'low' (hyperplasia score $<0.06)$ areas. Within an individual pancreas, hyperplasia score in any high area was significantly (paired $t$ test $\mathrm{p}<0.01$ ) greater than that in any low area. Hyperplasia scores in the pancreatic head (area B) had a lower correlation with adjacent areas in the head $($ area $\mathrm{A}, \mathrm{r}=0.35, \mathrm{p}<0.0001$; area $\mathrm{C}, \mathrm{r}=0.50$, $\mathrm{p}<0.0001)$ than with the uncinate process (area $\mathrm{D}$, $r=0.55, p<0.0001)$ or the tip of the pancreatic tail (area $\mathrm{J}, \mathrm{r}=0 \cdot 61, \mathrm{p}<0 \cdot 0001$ ).

EFFECT OF VARIATION IN PAPILLARY AND PANCREATIC DUCT ANATOMY

The number of specimens in each group, stratified by ampullary common channel size, was: $0 \mathrm{~mm}(26$ specimens), $<2 \mathrm{~mm}$ (29 specimens), 2-5 mm (30 specimens), $>5 \mathrm{~mm}$ (17 specimens). There was no significant difference in either prevalence of $\mathrm{DMH}$ or pancreas hyperplasia score between these groups. There was also no significant difference between these groups in prevalence of DMH or hyperplasia score, in tissue sections adjacent to the main papilla (area A).

The number of specimens within each category of pancreatic duct anatomy (Fig. 1, Table 1) was: I (30 specimens), II (three specimens), III (17 specimens), IV (four specimens), V (48 specimens). There was no significant difference in either prevalence of DMH or pancreas hyperplasia score between these categories of duct anatomy. The prevalence of DMH adjacent to the accessory papilla (area W, Fig. 1) was less (2/ $27,7 \%$ ) when the accessory papilla was patent (duct categories I, II, IV, Fig. 1) than $(13 / 49,27 \%)$ when the accessory duct stained with dye but the accessory papilla was not patent (duct category V), but this was not a significant difference ( $95 \%$ confidence). However the hyperplasia score adjacent to the accessory papilla (area W) was significantly less (Wilcoxon's test $p<0.05$ ) where the papilla was patent (duct categories I, II, IV) compared with where the papilla was not patent but the accessory duct communicated with the main duct (duct category V).

\section{Discussion}

The increased prevalence of DMH which has been reported in the head and neck of the pancreas compared with the body and tail ${ }^{16}$ would be anticipated if DMH was the result of reflux of either bile or duodenal contents into the pancreatic duct. Arrangements of the ampulla which could allow bile reflux through a 'common channel' were, however, not associated with increased prevalence of DMH. Nor was the prevalence greatest adjacent to the ampulla where duodenopancreatic reflux would be expected to be most marked.

There was a significant increase in DMH adjacent to the accessory papilla when it was occluded compared with when it was patent - suggesting that DMH adjacent to the accessory papilla may reduce its patency. The increased susceptibility to pain and pancreatitis associated with ventral unfused pancreas ${ }^{9}$ in which the majority of the pancreas drains through the accessory papilla, could be the result of increased pressure in the ventral pancreas ${ }^{12}$ produced by reduced drainage through a papilla which is obstructed by DMH. As would be expected from the reported prevalence, ${ }^{4}$ there were only four patients with ventral unfused pancreas (duct category IV) in the present study. Pancreas from these patients was not associated with significant increase in pancreas hyperplasia score. Although the incidence of pancreatitis is increased about six-fold in ventral unfused pancreas compared to pancreas with a fused duct system, " not all cases are affected by pancreatitis. The extent to which the minor duct changes of pancreatitis seen at ERCP correspond to the DMH measured in this study is not clear. ${ }^{13}$ The $70 \%$ prevalence of minor duct change seen at ERCP in ventral unfused pancreas, ${ }^{14}$ however, should be 


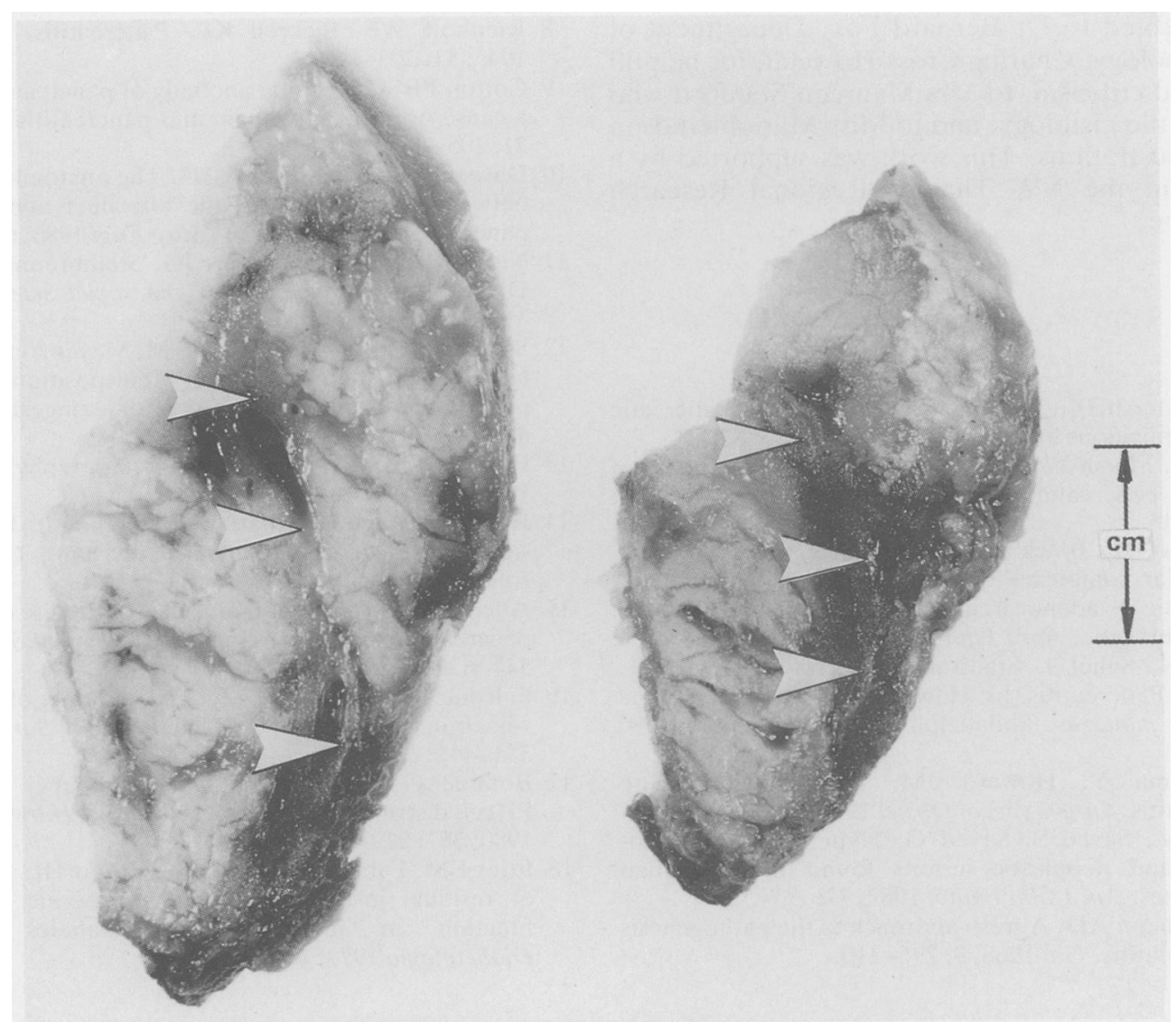

Fig. 3 Two sagittal slices through the pancreatic head. The slice on the right is closer to the duodenum. A septum is present, separating the dorsal (above and in front in each slice) from the ventral (below and behind) embryological component of the pancreatic head.

interpreted with caution in view of the $62 \%$ prevalence of DMH found overall and the absence of any increased prevalence of DMH in the four cases of ventral unfused pancreas. These minor histological abnormalities are not uncommon in normal pancreas and they become more common with age.'

While DMH was increased in the head and neck compared with the body and tail of the pancreas, on closer examination it had a patchy distribution which was different from that which would be produced by reflux, or by occlusion of a particular pancreatic segment. In addition to the head of the pancreas, the greatest amount of DMH was found in the uncinate process and the tip of the pancreatic tail. It is possible that this patchy distribution was the result of a variety of different factors, each affecting a separate area of the pancreas. Against this was that the correlation between amount of DMH seen in the pancreatic head with adjacent areas was not greater than that between head and uncinate process, or head and pancreatic tail. This would be more consistent with a response to the same agent acting throughout the pancreas.

These findings support the view that $\mathrm{DMH}$ is produced by external agents which injure the pancreas thereby stimulating duct and acinar cell proliferation. ${ }^{1}$ The patchy distribution noted in this study suggests that some areas of the pancreas are more vulnerable than others to this injury. Although the distribution of ducts of different diameter ${ }^{15}$ and of pancreatic blood supply ${ }^{16}$ is relatively uniform, the pancreas is not functionally uniform throughout - for example, there are differences in production of endocrine peptides within different areas. ${ }^{17}$ This may extend to exocrine function since the functions of endocrine and exocrine components of the pancreas are inter-dependent. ${ }^{18}$ Local variation in vulnerability to DMH could result from local differences in exocrine secretion, flow rate or duct juice composition. 
I am indebted to Dr Bernard Fox, Department of Histopathology, Charing Cross Hospital, for helpful advice and criticism, to Mrs Maureen Stanford who prepared the histology, and to Miss Marion Hudson for the illustrations. This work was supported by a grant from the NW Thames Regional Research Committee.

\section{References}

1 Allen-Mersh TG. What is the significance of pancreatic ductal mucinous hyperplasia? Gut 1985; 26: 825-33.

2 Parsa I. Marsh WL. Sutton AL. An in vitro model of human pancreatic carcinogenesis. Cancer 1981; 47: 1543-51.

3 Bockman DE, Black O, Mills LR, Webster PD. Origin of tubular complexes developing during induction of pancreatic adenocarcinoma by 7-12-dimethylbenzanthracene. Am J Pathol 1978; 90: 645-58.

4 Sarles H. Sahel J. Staub JL. Bourry J. Laugier R. Chronic Pancreatitis. In: Howat HT, Sarles H, eds. The exocrine pancreas. Philadelphia: WB Saunders, 1979: 402-40.

5 Nakashima Y, Howard JM. Drug-induced acute pancreatitis. Surg Gynecol Obstet 1977; 145: 105-9.

6 Pour PM. Sayed S. Sayed G. Hyperplastic, preneoplastic and neoplastic lesions found in 83 human pancreases. Am J Clin Pathol 1982; 77: 137-52.

7 McCutcheon AD. A fresh approach to the pathogenesis of pancreatitis. Gut 1968; 9: 296-310.
8 Rienhoff WF. Pickrell KL. Pancreatitis. Arch Surg 1945; 51: 205-19.

9 Cotton PB. Congenital anomaly of pancreas divisum as a cause of obstructive pain and pancreatitis. Gut 1980; 21: 105-14.

10 Dawson PR. Allen-Mersh TG. The anatomical relation between the retropancreatic bile duct and the main pancreatic duct. Ann R Coll Surg Engl 1983; 65: 188-91.

11 Nie NH, Hull CH, Jenkins JG, Steinbrenner K, Bent DH. Statistical package for the social sciences. Ncw York: McGraw Hill, 1975.

12 Staritz M. Hutteroth T, Manns M. Mayer zum Buschenfelde K-H, Pancreas divisum: predisposition to chronic pancreatitis due to chronic secretory congestion? Disch Med Wochenschr 1986; 111: 421-3.

13 Birnstingl MA. A study of pancreatography. Br J Surg 1959; 47: 128-39.

14 Rosch W, Koch H. Schaffer O, Demling L. The clinical significance of the pancreas divisum. Gastrointest Endosc 1976; 22: 206-7.

15 Allen-Mersh TG. The significance of the site of origin of pancreatic exocrine carcinoma. J Clin Pathol 1982; 35: 544-6.

16 Falconer CWA, Griffiths E. The anatomy of the blood vessels in the region of the pancreas. Br J Surg 1950; 37: 334-44.

17 Bommer G, Friedl U, Heitz PU, Kloppel G. Pancreatic PP cell distribution and hyperplasia. Virchows Arch [A] 1980; 387: 319-31.

18 Frier BM, Faber OK, Binder C, Elliott HL. The effect of residual insulin secretion on exocrine pancreatic function in juvenile onset diabetes mellitus. Diabetologia 1978; 14: 301-4. 